\title{
PUBG WINNER PLACEMENT PREDICTION USING ARTIFICIAL NEURAL NETWORK
}

\author{
Madhurya Manjunath Mamulpet \\ Faculty of Engineering, Environment and Computing, Coventry University \\ MSc. Data Science and Computational Intelligence (ECT104) Stage 1 \\ Coventry, United Kingdom
}

\begin{abstract}
The main objective is to predict the placement ranking of the player in PUBG (Players unknown battlegrounds) using the player's position and stats of data. PUBG is a game that has turned out to be famous in recent years. For this diversion, the last position is the most essential marker of the player's capacity. This task centres on anticipating the last position and finding ideal techniques of the diversion. With information from PUBG, we apply a few Artificial Neural Network techniques including Deep Learning, light GBM model, and MLP regression model to make the winner predictions.
\end{abstract}

Keyword: Deep learning, LGBM, MLP.

\section{INTRODUCTION}

Player Unknown Battle Grounds is a multiplayer online battle game i.e. developed by the PUBG Corporation. This is a game where 100 players fight or play in battle with each other. Players can choose to fight alone, with a group of 2 or 4 . Although, the size of the team can be modifiable through featured engineering. The winner of the game will be the player that survived or stays alive till the end of the game. This can be through various strategies such as killing an opponent using a weapon or surviving by healing or hiding. There are many tools in a game that helps the player to kill his enemies like armour, weapons, healing kits, vehicles and many other resources. The dataset contains five million records out of which there are 28 features(1). The main objective is to predict the player winning percentage based on various aspects such as the number of kills obtained, survival rates, number of players alive and many more variables. This is done using an algorithm the players win percentage will be ranged between 0 1. These problems are solved through analysing data and feature extraction technique upon which the winning percentage depends on.

The techniques used on the player records to analyse and extract are neural network MLP Regression, Light GBM, Deep Learning, Tensor flow etc. The accuracy of the model is validated and the result obtained reached the desired requirement.

This paper is presented in the following order.

- Related work that is researched on the same data as well as a similar game prediction.

- The methodology used for placement prediction

- Data description with the explanation of their features

- EDA data analysis

- Experimental Setup the process explanation to perform validation on data

- Results obtained by the applied methods

- The conclusion of the overall research.

- Ethical, legal and Social Issues

- Bibliography

\section{RELATED WORK}

The researchers have used the same data set to predict the final $\operatorname{rank}(2)$. They trained the model on various techniques such as regression and tree models which also provided optimal strategy. The model trained used the linear regression, ridge regression and LGBM technique the models were tuned and the 5-fold cross was applied to validate the data. LGBM aggregates the GBDT with GOSS algorithm and EFB. Mathematically represented as

$\tilde{V}_{j}(d)=\frac{1}{n}\left(\frac{\left(\sum_{x_{i} \in A_{l}} g_{i}+\frac{1-a}{b} \sum_{x_{i} \in B_{l}} g_{i}\right)^{2}}{n_{l}^{j}(d)}+\frac{\left(\sum_{x_{i} \in A_{r}} g_{i}+\frac{1-a}{b} \sum_{x_{i} \in B_{r}} g_{i}\right)^{2}}{n_{r}^{j}(d)}\right)$.

Equation 1: LGBM Computation formula

In particular, there is also a semi-supervised combination of Gaussian method used with generalized k-mean clustering to aggregate informal information.

The results Obtained with an MAE of 0.0204 which was ranked 57 in the leader board of the competition. That also concludes that the LGBM using a learning rate of 0.05 was faster, lighter and best fit model. 
International Journal of Engineering Applied Sciences and Technology, 2019

Vol. 3, Issue 12, ISSN No. 2455-2143, Pages 107-118

Published Online April 2019 in IJEAST (http://www.ijeast.com)

\begin{tabular}{|c|c|c|c|}
\hline MAE on 20\% validation set & Linear Regression & Ridge Regression & Light GBMI \\
\hline \hline Raw features & 0.09000 & 0.08989 & 0.056654 \\
\hline Raw + mean & 0.05736 & 0.05736 & 0.04158 \\
\hline Raw + mean + sum + max - min & 0.04845 & 0.04845 & 0.02896 \\
\hline Everything above + match_mean + size & 0.04825 & 0.04825 & 0.02755 \\
\hline Everything above + cross validation & 0.04812 & 0.04810 & 0.0204 \\
\hline
\end{tabular}

Table 1: Results Obtained

Similarly, Researchers have been trying to predict the winner of the game of football with many data science and mathematical techniques. This paper (3) predicts the winning team in the NFL American football team using neural network deep learning techniques. According to the game, the team of two will have a ball that must be put to either side of the net which is assigned to each of the time the goal is to predict which team can do it first. They also used probability technique to see the ranking of each player with their strength. In this case, they were able to correctly classify the data with $98 \%$ using many to one method. Also used an LSTM technique architecture. The data consists of 13 datasets with 130 features in the game. Using the following methods the researcher has trained and validated the dataset. The ANN output predicts the instance of training there can be a classification wrongly predicted to minimize that a cross entropy is used by doing this we can apply the back propagation method. For this model RNN i.e. recurrent Neural Network with a t-1 output this is helpful for predicting the NFL-game that helps in knowing which team often wins. Many to one classification are used to predict a binary classification. LSTM is used for dependencies of the long term that is better than RNN they include batch size, time, features as components for input format for example time of steps is determined by the data passed RNN uses kfolds of 10 to train the data set both RNN and LSTM use a 4 layer model. All the testing for deep learning was trained for a 4 layer architecture in deep learning with a Relu activation function parameter and if optimizer which has a better performance. The results obtained from all the models do not have much difference, However, they have concluded that LSTM is the best fit compared to any other model with an accuracy of $63.31 \%$ this model is robust and can outperform for predicting.

\begin{tabular}{l|l|l|l|}
\multicolumn{1}{l}{ Model } & \multicolumn{1}{c}{ Optimal parameter } & \multicolumn{1}{c}{ Accuracy $(\%)$} & 95\% confidence interval \\
\hline ANN & $\begin{array}{l}\text { Structure: }(2,2,2) \\
\text { Activation: tanh }\end{array}$ & 61.73 & {$[0.56,0.68]$} \\
\cline { 2 - 4 } & $\begin{array}{l}\text { Structure: }(5,5,5) \\
\text { Batch size: } 1790 \\
\text { Activation: } \tanh \end{array}$ & 63.31 & {$[0.61,0.66]$} \\
\cline { 2 - 4 } & $\begin{array}{l}\text { Structure: }(25,25,25) \\
\text { Batch size: } 1790 \\
\text { Activation: tanh }\end{array}$ & 62.05 & {$[0.57,0.67]$} \\
\hline
\end{tabular}

Figure 1: Results obtained

\section{METHODOLOGY}

\section{i) Light GBM (Gradient Boost Machine)}

It's fast and high-speed Gradient framework with a new algorithm which is subsided from decision tree algorithms that divides the data depth or widthwise instead of leaf wise(4). The LGBM can decrease the level order by using the same leaf wise algorithm and results are more effective and improvised that can boost the current existing algorithm.

\begin{tabular}{|l|l|}
\hline Learning Rate & 0.1 \\
\hline N_Estimators & 50,250 \\
\hline Number of leaf & 200 \\
\hline Boosting Type & Gbdt, dart, goss, of \\
\hline
\end{tabular}

Table 2: LGBM Parameters

When the model is trained through the deep learning data is optimised using the Adams learning rate which is 0.01

The benefits of using this model are

- It is fast in training data and uses less memory

- Provides better compatibility with large data

- It has improvised the accuracy by using the gradient boosting algorithm

According to our model, the main objective is to reduce the function loss which means the MSE i.e. The mean squared error. Gradient boosting model helps in minimizing the loss and to find the predicted value it takes alpha as the learning rate. The best parameters used for this model is learning rate at 0.3 $\mathrm{N}$ estimator with a best of 250 iterations and there is 200 leaf node.

\section{ii) Deep Learning}

The neural system is made out of three layers, to be specific information layer, concealed layer and the yielding layer. The actuation work is utilized to locate the weighted contribution of each unit in the layers. Hyperparameter tuning is done to accomplish demonstrate enhancement. Utilised Keras library to enable us to prepare the best model for our information(5). Deep learning is a neural network comprising of progressive layers in which each layer later changes the data into more unique representation. In deep learning adapting more layers, means higher learning levels of the model. The output layer consolidates all the highlights and makes an assumption. Thus it contrasts from Neural Network. While straightforward Neural Network utilizes just a single concealed layer which isn't reasonable for learning complex highlights, deep 


\section{International Journal of Engineering Applied Sciences and Technology, 2019 \\ Vol. 3, Issue 12, ISSN No. 2455-2143, Pages 107-118 \\ Published Online April 2019 in IJEAST (http://www.ijeast.com)}

learning utilizes various concealed layers to become familiar with these intricate highlights which may hazard overfitting. To keep away from this, we use batch normalization which standardizes and scales the yield of the enactment at each layer to keep away from esteems heading off to the limits. This enables each layer to gain uniquely in contrast to different layers, and thus abstains from overfitting. It also has the impact of diminishing the preparation time. We moreover use dropout at each concealed layer to abstain from overfitting by overlooking a set measure of neurons yield at each layer. Thus, deep learning can be extravagant and requires huge dataset to prepare itself on.

\begin{tabular}{|c|c|c|}
\hline \multirow{2}{*}{\multicolumn{3}{|c|}{$\begin{array}{l}\text { Layer (type) } \\
=============1 \text { Output Shape }\end{array}$}} \\
\hline & & \\
\hline dense_5 (Dense) & (None, 512) & 22528 \\
\hline batch_normalization_4 (Batch & (None, 512) & 2048 \\
\hline dropout_4 (Dropout) & (None, 512) & 0 \\
\hline dense_6 (Dense) & (None, 256) & 131328 \\
\hline batch_normalization_5 (Batch & (None, 256) & 1024 \\
\hline dropout_5 (Dropout) & (None, 256) & $\theta$ \\
\hline dense_7 (Dense) & (None, 128) & 32896 \\
\hline batch_normalization_6 (Batch & (None, 128) & 512 \\
\hline dropout_6 (Dropout) & (None, 128) & $\theta$ \\
\hline $\begin{array}{l}\text { dense_8 (Dense) } \\
==========================0\end{array}$ & $\begin{array}{l}\text { (None, 1) } \\
============\end{array}$ & 129 \\
\hline $\begin{array}{l}\text { Total params: } 190,465 \\
\text { Trainable params: } 188,673 \\
\text { Non-trainable params: } 1,792\end{array}$ & & \\
\hline
\end{tabular}

Figure 2: Deep learning Model Architecture.

To specify the parameters used in deep learning model architecture depicted in the above figure. We set the epoch to 20 i.e. the number of time the data cycle runs. The higher the number of times the better improvement in the model and there are 4 hidden layers on the normalisation of which the optimizer uses a learning rate of 0.01 and epsilon is used to reduce the error that prevents from diving by zero, and decay is the weight used for the optimisation

$$
f(x)=\max (0, x)
$$

Equation 2: Deep Learning

Relu Rectified linear unit function that permits the activation function used for network design in the hidden layer to input is derived from its domain and the sigmoidal function for its output.

\section{iii) MLP Regression}

Multilayer Perceptron Regression model is similar to Logistic regression in which the inputs are changed by a non-linear function(6). MLP is a supervised learning method which is known as back-propagation for dataset training through multiple layers and utilises the nonlinear transformation which differs from linear perceptron. The data cannot be linearly separable. The primary objective of non-direct relapse is to give an estimation of the genuine parameter on account of perceptions $((\mathrm{x} 1, \mathrm{y} 1), \cdot . \cdot$, (in, in)). This model is used for predicting the inputs when real-value quantity is predicted this should be possible by limiting the MSE work. The file type is csv text data for input. According to math in MLP they are able to approximate an XOR operator with other non-linear function. Here there is also a boundary error rate where the data execution continues no longer than this. This stat is called convergence.

$$
\begin{aligned}
& y=\varphi\left(\sum_{i=1}^{n} w_{i} x_{i}+b\right)=\varphi\left(\mathbf{w}^{T} \mathbf{x}+b\right) \\
& \text { w: weights of vector } \\
& \text { x: vector inputs } \\
& \text { B: Bias; phi non-linear activation function }
\end{aligned}
$$

Equation 3: MLP Function

\section{DATA DESCRIPTION}

\begin{tabular}{|c|l|l|}
\hline & \multicolumn{1}{|c|}{ Attribute } & \multicolumn{1}{|c|}{ Definition } \\
\hline 1 & Player ID & Identification number given to each player \\
\hline 2 & Group ID & Identification number given to each group \\
\hline 3 & Boosts & Total boost items used \\
\hline 4 & Headshot Kills & Number of enemies killed by a direct headshot \\
\hline 5 & Kill Assist/Assist & Enemy players knocked out that were killed by teammates \\
\hline 6 & Damage Dealt & Total damage dealt by the player \\
\hline 7 & DBNO & Total enemies knocked out \\
\hline 8 & Heals no & Total healing items used \\
\hline 9 & Kill place ranking & Player ranking based on total enemies killed \\
\hline 10 & Kill points & Kill based overall ranking of player \\
\hline 11 & Kill streak & Total enemies killed by player in a short amount of time \\
\hline 12 & Kills & Number of enemy players killed in a game \\
\hline 13 & Longest kill & Longest distance between player and enemy killed \\
\hline 14 & Num groups & Total groups in a match \\
\hline 15 & Match duration & Time duration of a match in seconds \\
\hline 16 & Match Id & Identification number given to a match \\
\hline 17 & Match type & Type of match (solo,duo,squad,arcade, etc..) \\
\hline 18 & Max place & The maximum rank a team player got in the match \\
\hline 19 & Rank points & Elo like ranking of player \\
\hline 20 & Revives & Number of times a player revives his team mates \\
\hline 21 & Ride distance & Total distance covered by riding/driving vehicles \\
\hline 22 & Road-kills & Number of enemies killed while driving \\
\hline 23 & Swim distance & Total distance covered by swimming \\
\hline 24 & Team kills & Total number of teammates killed \\
\hline 25 & Vehicle destroys & Total number of vehicles destroyed \\
\hline 26 & Walk distance & Total distance covered by walking or running \\
\hline 27 & Weapons acquired & Total number of weapons acquired \\
\hline 28 & Win points & Score based on overall ranking of player \\
\hline 29 & Win place perc & The winning percentage/probability of the player \\
\hline
\end{tabular}

Table 3: Data Description

\section{DATA ANALYSIS \& FEATURE ENGINEERING}

The data analysis pattern can be determined by knowing the feature usages, this leads us to apply 


\section{International Journal of Engineering Applied Sciences and Technology, 2019 Vol. 3, Issue 12, ISSN No. 2455-2143, Pages 107-118 \\ Published Online April 2019 in IJEAST (http://www.ijeast.com)}

EDA Exploratory Data Analysis alludes to the basic procedure of performing introductory examinations on data to find patterns, to spot anomalies, to test speculation and to check presumptions with the assistance of synopsis measurements and graphical presentation(7). By analyzing the data we could generate a new set of a feature from the existing one that could help in improvising our model. So we find the total number of players by their match id and group id, we can find the total distance by combining the ride distance, swim distance, walking distance and many more(8). Shown below that contribute to evaluating the prediction efficiently.

\begin{tabular}{|l|l|}
\hline \multicolumn{1}{|c|}{ Features } & \multicolumn{1}{|c|}{ Explanation } \\
\hline totalPlayers & Total Players in the match \\
\hline teamSize & $\begin{array}{l}\text { Total team member in a } \\
\text { team } \\
\text { Match type like solo, } \\
\text { duo,quad etc. } \\
\text { normMatchType }\end{array}$ \\
\hline totalDistance & Total kills by team \\
\hline maxPossiblekills & boost+heals+weapon \\
\hline itemsUsed & Figure $3:$ Feature Engineering \\
\hline
\end{tabular}

When we look into the data distribution pattern there are some players who score exactly 0 or 1 , the rest are in between the range. We shall assume that on an average the winning percentage per match is 0.5 (mean) but this cannot be same for all the matches which depict on a lower average this happens due to players quitting the game before it ends. The correlation that we found between these attributes is as follows.

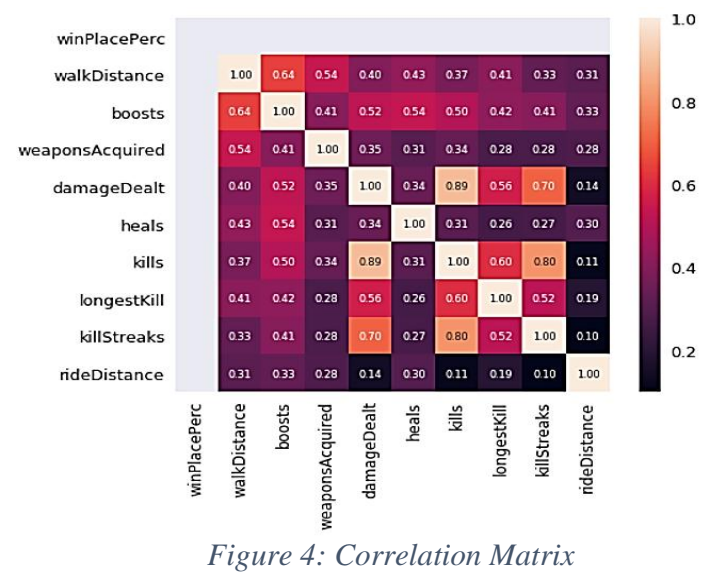

\section{EXPERIMENTAL SETUP}

The data is taken from kaggle PUBG prediction placement competition with nearly five million datasets out of which 1.3 million datasets are trained and tested with 29 attributes present the training and testing validation is split in the ratio of $7: 3(9)$.
Jupyter Notebook is used for coding with libraries such as sci-kit learn, numpy and Keras Tensor flow Backend is used for hierarchical deep learning models. Performed on a windows 8 computer. Tensor flow is used to control structure and it is an open source by Google. The data frame is iterated through all the columns of a data frame and the datatype is altered to reduce the memory usage. The dataset is loaded from a CSV file. Once this is done we try to find the Data Distribution pattern and correlation by plotting appropriate graphs.

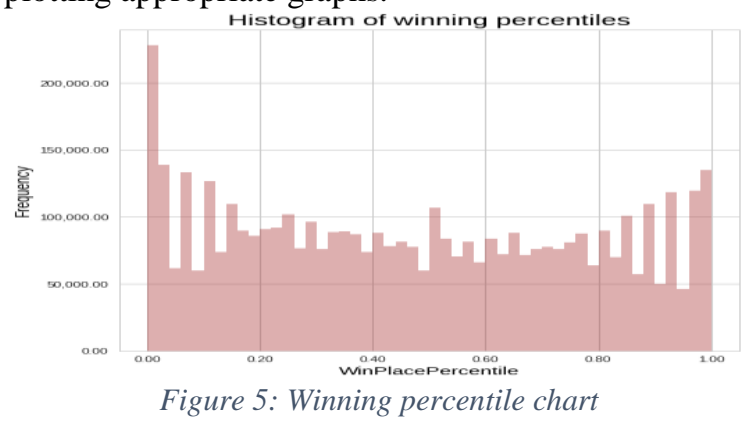

The data set must be filtered i.e. done by dropping the columns or records which are 0 or no value which helps in preventing the error and reduces memory usage. Now feature engineering is done by assessing the numeric value for generated correlated new features which was previously discussed in the Feature engineering section. This new match consists of features like solo, duo and squad which depicts the size of the team.

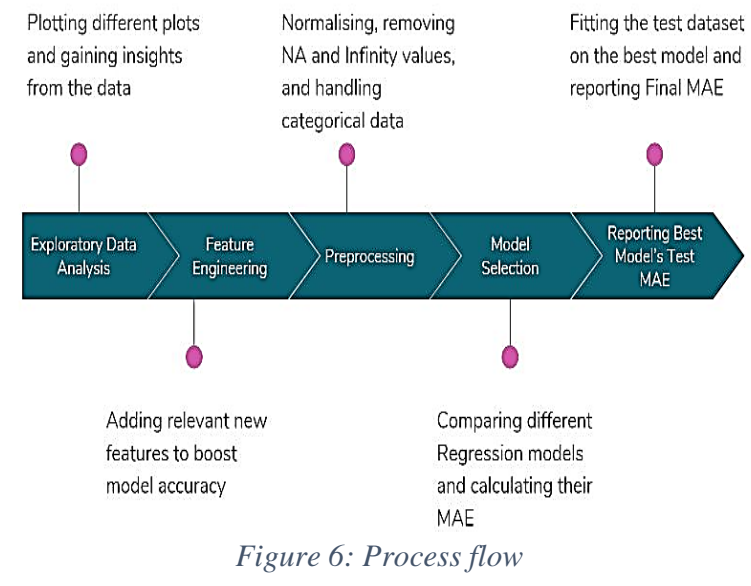

We construct the model LGBM Regression. A regression model is used than classification as there are independent variables that are correlated to dependent data this method is used to find the relation between the data. Deep reinforcement learning methods were not easy to use as that requires high computational power and consumed a lot of time. Hence using these methods to compensate and reduce memory consumption and compilation time. 
The initialisation of the simple MLP takes places by imparting training and validating the data with appropriate parameters than by facing a loss the next model i.e. the deep learning technique uses batch normalisation to handle the loss of data by using more layers and an activation function which then iterates at its best of 200 with a 20 epoch and early stop set to 10. Lastly, the LGBM model is initialized first experimented with a grid search that took endless run time then switched to this technique that took 222 seconds of run time with best parameters and a learning rate of 0.05

\section{RESULTS}

The accuracy of all the models trained and tested are shown as below:

\begin{tabular}{|c|c|c|c|}
\hline Model & LGBM & $\begin{array}{l}\text { Deep } \\
\text { Learning }\end{array}$ & MLP \\
\hline MAE & 0.0539 & 0.05947 & 0.08525 \\
\hline $\mathbf{R}^{2}$ & $93.6 \%$ & $90.61 \%$ & $85.4 \%$ \\
\hline
\end{tabular}

The obtained result shows a 5\% improvisation between the MLP and deep learning model. Here MLPs learning rate is Adaptive. The first time the improvement of the model was stagnant as the model stopped running after 2 continuous epochs which mean the loss of the data which stopped at best 14 iterations. The Mae was better obtained.

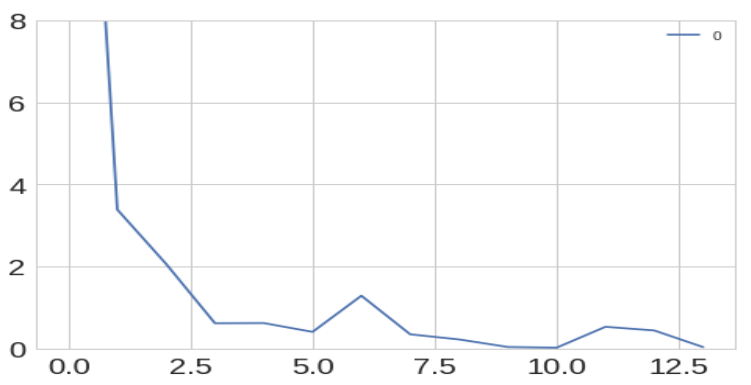

Figure 7: Loss Curve MLP

When the model was trained on deep learning we used the previous loss of data results to compare with the new value the kernel initialization was normal and used relu activation and sigmoid activation function after batch normalization of data to remove the unwanted data. Here epsilon is $1 \mathrm{e}-4$ used to prevent from 0 division errors. The model tested on deep learning gave a satisfying result.

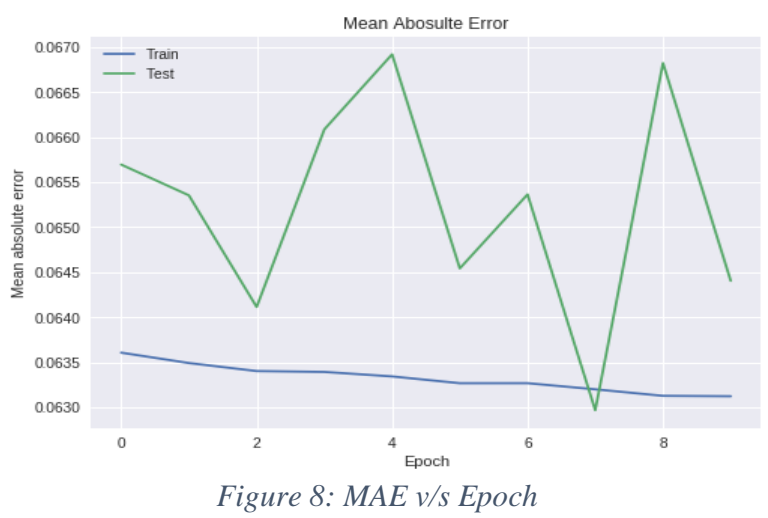

This above graph compares the data error rate and iteration rate the green line is the tested data and blue for trained data.

LBGM model that satisfied all the conditions with the best fit for data, among all the other models this model used less memory, it was fast for a large dataset and the best iterations done was 250 with a random state that no other model could do with the training of data. With a speed of 222 seconds which is instantaneous, it predicts the winning percentage of a player in pubg game.

The graph states the parameters used with $n$ estimators of 250 between the mean absolute error which is set to early stop rounds of 10 which means that before it reaches the max value it's self its stops with a leaf node of 200. The overall conclusion states the validation applied to the dataset this model gave the highest MAE with 0.0539 .

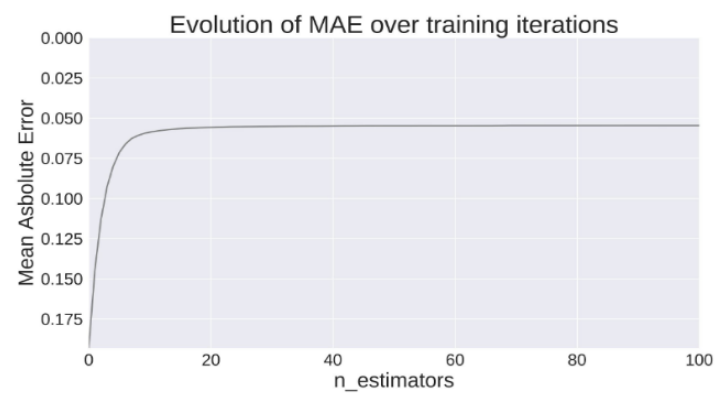

Figure 9: LGBM MAE estimators

\section{CONCLUSION}

The results obtained from the research are significantly strong to support the strategies in which a player can play in the battlefield with various attributes that contribute towards obtaining the winning percentile. The various comparison attributes to consider for the winning players is depicted in the graph as follows 


\section{International Journal of Engineering Applied Sciences and Technology, 2019 Vol. 3, Issue 12, ISSN No. 2455-2143, Pages 107-118 \\ Published Online April 2019 in IJEAST (http://www.ijeast.com)}

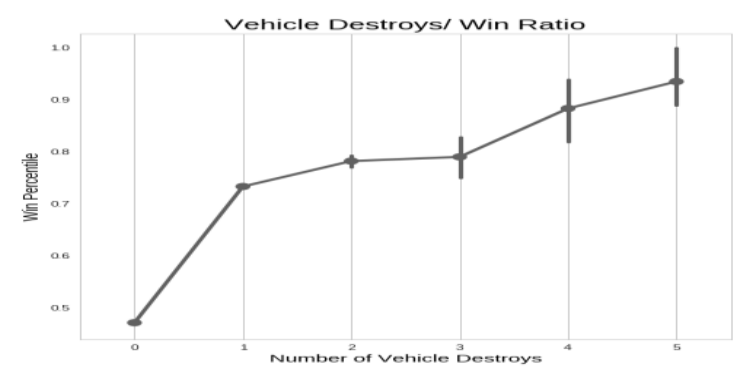

Figure 10: Winning percentile based on No of Kills

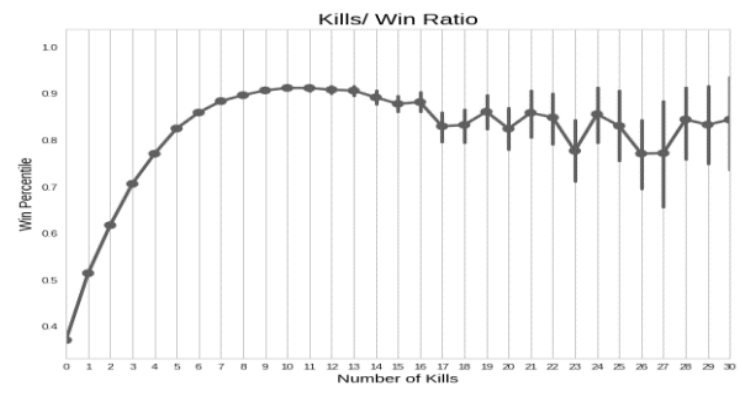

Figure 11: Winning percentile based on No of Vehicles destroyed

We have also discovered that the size of the team matters for predicting the winning percentile and in fewer cases the accuracy of the prediction goes high in which the case is where the player would have ended the game before it would have reached the end that gives us a wrong assumption. Further when we test the data records of the players or users playing the game worldwide its more than millions of people we used their records to predict the winner by using few methodologies out if which we have succeeded with all the three models that were successfully implemented with a better run time. LGBM model outstands with better improvement in the model, reduction in memory usage and faster run time with a large amount of dataset with an accuracy of $93 \%$ best fit model. Next, with a very less difference deep learning method in neural networks was trained and tested on the data using batch normalization helped in recovering the data loss which had occurred in MLP with a Lower accuracy of $85 \%$ and fewer iterations. Further, we can also conclude that a player can also win by hiding in the safe zones in the game(10).

\section{ETHICAL, LEGAL AND SOCIAL ISSUES}

The technology information for this particular area consists various ethical(11), legal and social issues many user playing on PC or Xbox have been reduced drastically as they have identified software developed bugs in the PUBG Road map and quality of life issue with the game for example player holding a weapon while moving close to rock under water that must be fixed. The game is poorly optimized on a powerful computation. As far as the legal issue concerns (12) there was a complain file against a pubg corporation that specifies about the copyrights of a similar game known as fortunate.

\section{REFERENCE}

1. Hodge V, Devlin S, Sephton N, Block F, Drachen A, Cowling P. Win Prediction in Esports: Mixed-Rank Match Prediction in Multi-player Online Battle Arena Games. 2017 Nov 17 [cited 2019 Mar 30]; Available from: http://arxiv.org/abs/1711.06498

2. Wei W, Lu X, Li Y. PUBG: A Guide to Free Chicken Dinner [Internet]. 2018 [cited 2019 Mar 30]. Available from: http://cs229.stanford.edu/proj2018/report/127 .pdf

3. Bosch P, Bhulai S. Predicting the winner of NFL-games using Machine and Deep Learning [Internet]. 2018 [cited 2019 Mar 30]. Available from: https://beta.vu.nl/nl/Images/werkstukbosch_tcm235-888637.pdf

4. What is LightGBM, How to implement it? How to fine-tune the parameters? [Internet]. [cited 2019 Mar 30]. Available from: https://medium.com/@pushkarmandot/httpsmedium-com-pushkarmandot-what-islightgbm-how-to-implement-it-how-to-finetune-the-parameters-60347819b7fc

5. Lets Play-A "PUBG" Dataset step by step tutorial - Towards Data Science [Internet]. [cited 2019 Mar 30]. Available from: https://towardsdatascience.com/lets-play-apubg-step-by-step-tutorial-1c0b38b322e8

6. Adler J. On using Artificial Neural Network models to predict game outcomes in Dota 2 VIKTOR WIDIN [Internet]. DEGREE PROJECT TECHNOLOGY. 2017 [cited 2019 Mar 30]. Available from: https://pdfs.semanticscholar.org/796c/8a7a65 5f5bdeab7084baca2fa0b9a4f6a01e.pdf

7. Data Analysis of PlayerUnknown's Battlegrounds (PUBG) - Introduction \&amp; Data Preparation [Internet]. [cited 2019 Mar 30]. Available from: https://medium.com/@aliciali_7397/dataanalysis-of-playerunknowns-battlegroundspubg-introduction-data-preparation94da7b64614e

8. RPubs - PUBG Placement Prediction EDA [Internet]. [cited 2019 Mar 30]. Available from: https://rpubs.com/tianyi/pubgeda

9. PUBG Finish Placement Prediction (Kernels Only) | Kaggle [Internet]. [cited 2019 Mar 
International Journal of Engineering Applied Sciences and Technology, 2019 Vol. 3, Issue 12, ISSN No. 2455-2143, Pages 107-118

Published Online April 2019 in IJEAST (http://www.ijeast.com)

30]. Available from:

https://www.kaggle.com/c/pubg-finish-

placement-prediction/data

10. PUBG Data Analysis - Exploring

PLAYERUNKNOWN'S

BATTLEGROUNDS Statistics through Data

Science [Internet]. [cited 2019 Mar 30].

Available from:

https://pubganalysis.wordpress.com/

11. The first rule of Playerunknown's

Battlegrounds says a lot: stop the racial

harassment - The Verge [Internet]. [cited

2019 Mar 31]. Available from:

https://www.theverge.com/2017/8/3/1609319

0/playerunkown-battlegrounds-harassment

12. What We Know And What We Don't About

PUBG's Legal Fight With 'Fortnite' In Korea

[Internet]. [cited 2019 Mar 31]. Available

from:

https://www.forbes.com/sites/thomasbaker/20

18/06/05/what-we-know-and-what-we-dont-

about-pubgs-legal-fight-with-fortnite-in-

korea/\#31c9d7b23901 


\section{Appendix}

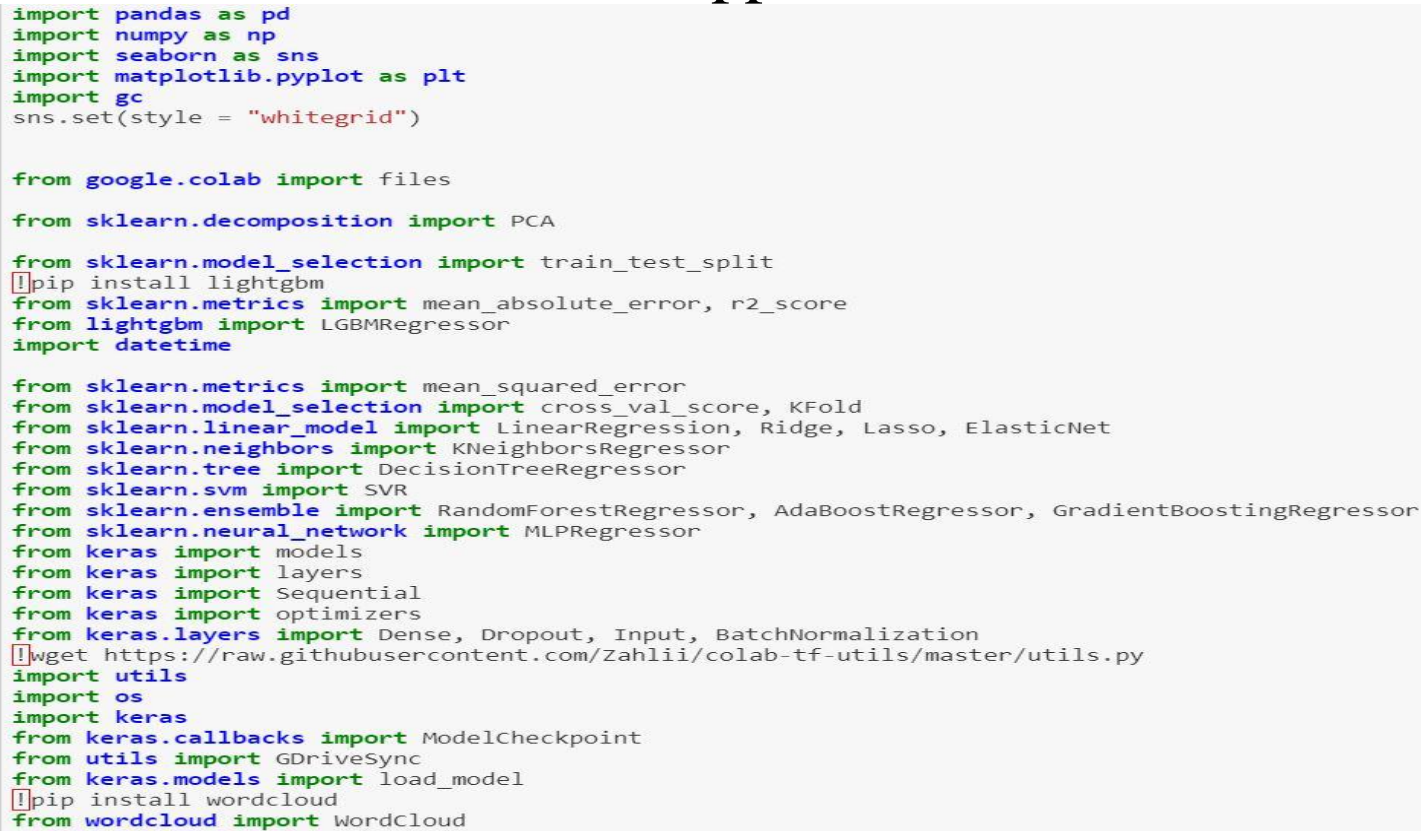

Figure 12:Import Libraries

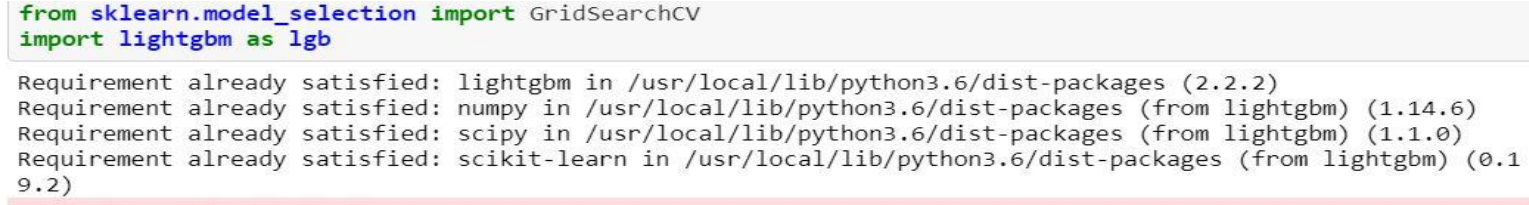

Figure 13: use of tensor flow

In [0]: train.drop(['match_mean', 'match_median'], axis=1)

\# data.drop('match_median')

train.columns

Out[0]: Index(['Id', 'groupId', 'matchId', 'assists', 'boosts', 'damageDealt', 'DBNOs',

'headshotKills', 'heals', 'killPlace', 'killPoints', 'kills',

'killStreaks', 'longestKill', 'matchDuration', 'matchType', 'maxplace',

'numGroups', 'rankPoints', 'revives', 'rideDistance', 'roadkills',

'swimDistance', 'teamKills', 'vehicleDestroys', 'walkDistance',

'weaponsAcquired', 'winPoints', 'winPlacePerc', 'match_mean',

'match_median'],

dtype='object' $)$

Figure 14: Dropping 0 value data 
International Journal of Engineering Applied Sciences and Technology, 2019

Vol. 3, Issue 12, ISSN No. 2455-2143, Pages 107-118

Published Online April 2019 in IJEAST (http://www.ijeast.com)

\begin{tabular}{|c|c|c|c|c|c|c|c|c|c|c|c|}
\hline \multirow[t]{2}{*}{ In $[\theta]$ : } & \multicolumn{11}{|c|}{ 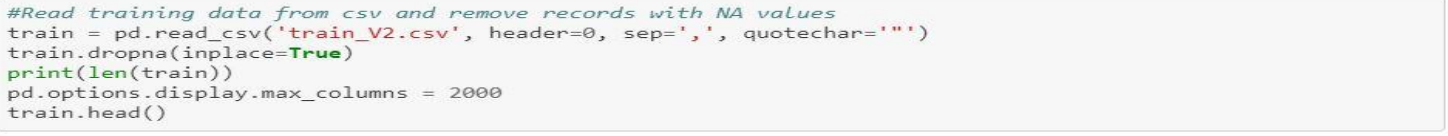 } \\
\hline & \multicolumn{11}{|c|}{4446965} \\
\hline \multirow[t]{7}{*}{ Out $[\theta]$ : } & & Id & groupld & matchld & assists & boosts & damageDealt & DBNOs & headshotKills & heals & killPla \\
\hline & 0 & 7 f96b2f878858a & $4 \mathrm{~d} 4 \mathrm{~b} 580 \mathrm{de} 459 \mathrm{be}$ & a10357fd1a4a91 & 0 & 0 & 0.00 & 0 & 0 & 0 & 60 \\
\hline & 1 & eef90569b9d03c & $684 d 5656442 \mathrm{f} 9 \mathrm{e}$ & $a e b 375 f c 57110 c$ & 0 & 0 & 91.47 & 0 & 0 & 0 & 57 \\
\hline & 2 & 1eaf90ac73de72 & $6 a 4 a 42 c 3245 a 74$ & 110163d8bb94ae & 1 & 0 & 68.00 & 0 & 0 & 0 & 47 \\
\hline & 3 & $4616 d 365 d d 2853$ & a930a9c79cd721 & f1f1f4ef $412 d 7 e$ & 0 & 0 & 32.90 & 0 & 0 & 0 & 75 \\
\hline & 4 & $315 c 96 c 26 c 9 a a c$ & de04010b3458dd & $6 \mathrm{dc} 8 \mathrm{ff} 871 \mathrm{e} 21 \mathrm{e} 6$ & 0 & 0 & 100.00 & 0 & 0 & 0 & 45 \\
\hline & \multicolumn{11}{|c|}{4} \\
\hline \multirow[t]{2}{*}{ In $[\theta]$ : } & \multicolumn{11}{|c|}{ 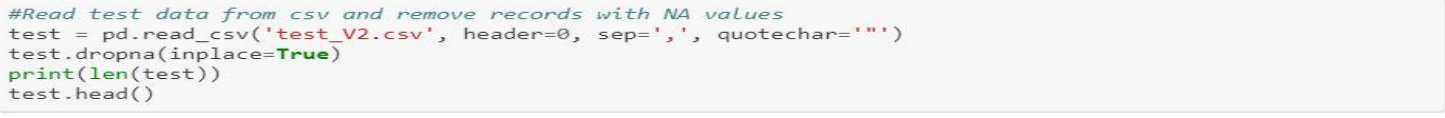 } \\
\hline & \multicolumn{11}{|c|}{1934174} \\
\hline \multirow[t]{6}{*}{ Out $[\theta]$ : } & & Id & groupld & matchld & assists & boosts & damageDealt & DBNOs & headshotKills & heals & killPla \\
\hline & 0 & $9329 \mathrm{eb} 41 \mathrm{e} 215 \mathrm{eb}$ & $676 b 23 c 24 e 70 d 6$ & 45b576ab7daa7f & 0 & 0 & 51.46 & 0 & 0 & 0 & 73 \\
\hline & 1 & 639bd0dcd7bda8 & $430933124148 d d$ & 42a9a0b906c928 & 0 & 4 & 179.10 & 0 & 0 & 2 & 11 \\
\hline & 2 & 63d5c8ef8dfe91 & Ob45f5db20ba99 & $87 e 7 e 4477 a 048 e$ & 1 & 0 & 23.40 & 0 & 0 & 4 & 49 \\
\hline & 3 & cf5b81422591d1 & b7497dbdc $77 f 4 a$ & 1b9a94f1af67f1 & 0 & 0 & 65.52 & 0 & 0 & 0 & 54 \\
\hline & 4 & ee6a295187ba21 & 6604ce20a1d230 & 40754a93016066 & 0 & 4 & 330.20 & 1 & 2 & 1 & 7 \\
\hline
\end{tabular}

Figure 15: Training and testing data

\section{LightGBM}

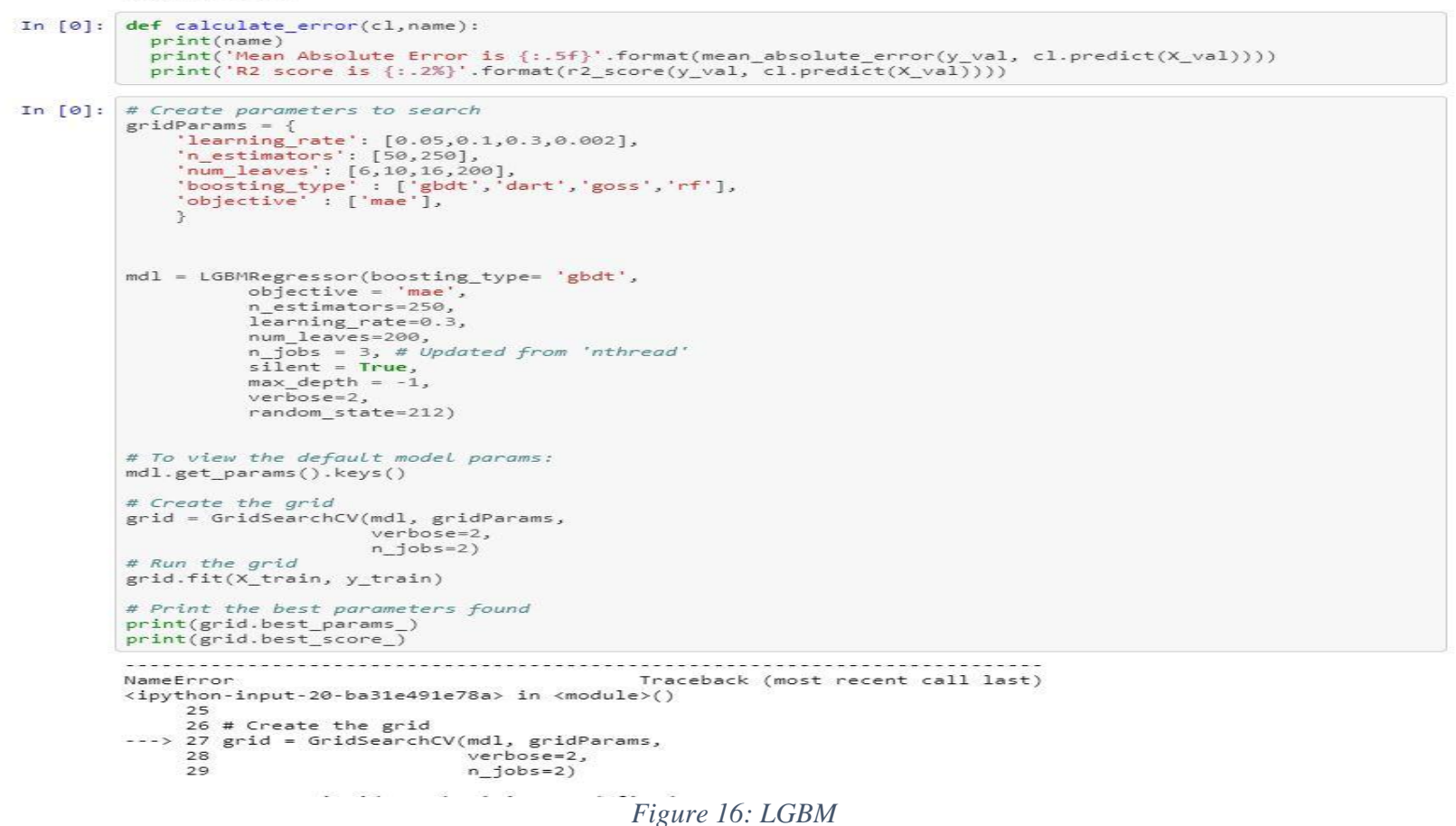

Figure 16: LGBM 
International Journal of Engineering Applied Sciences and Technology, 2019

Vol. 3, Issue 12, ISSN No. 2455-2143, Pages 107-118

Published Online April 2019 in IJEAST (http://www.ijeast.com)

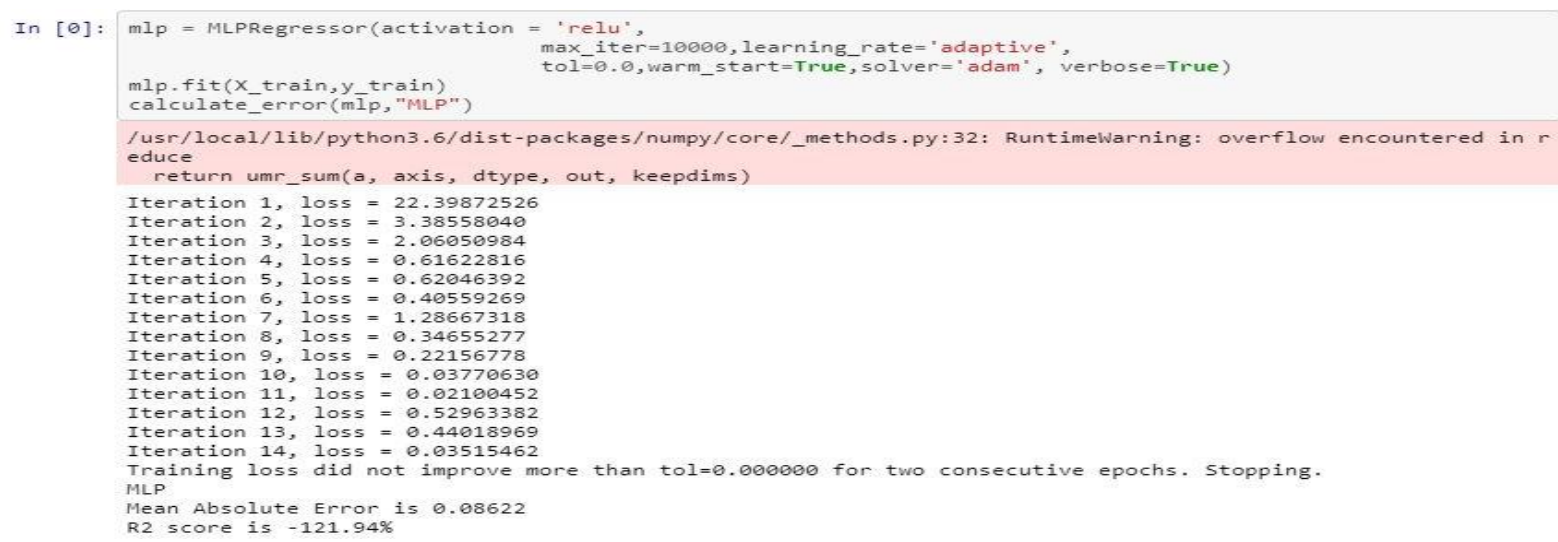

Figure 17: MLP

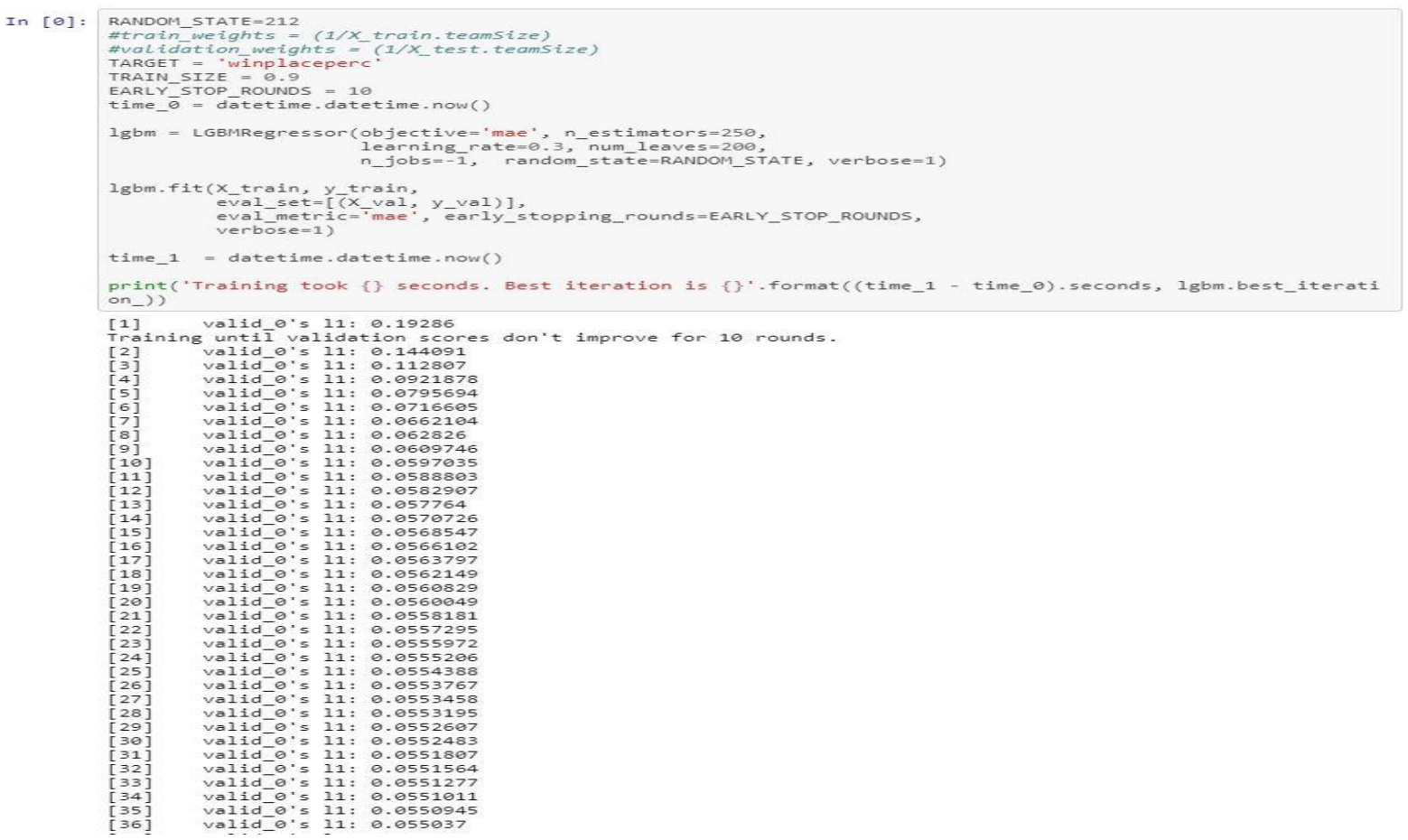

Figure 18: Random state iteration goes on till 250

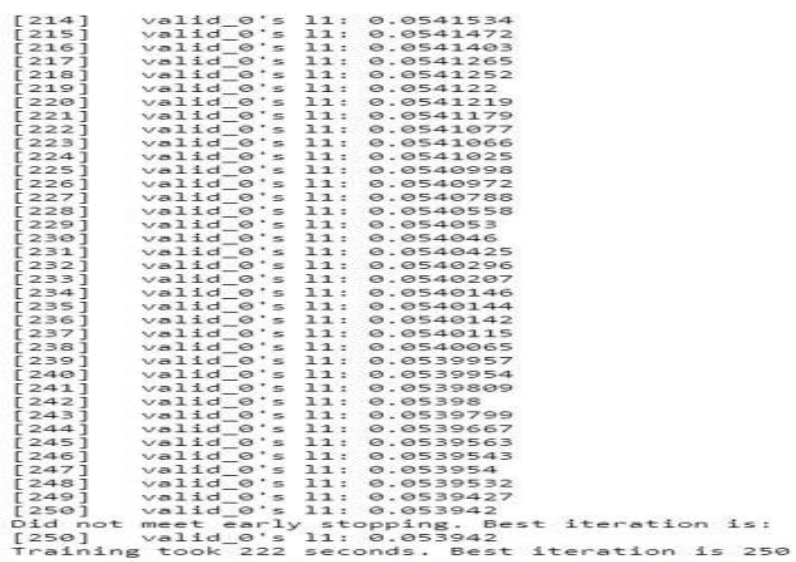

Figure 19: Validation of LGBM 


\section{Deep Learning}

In $[\ominus]$ : filepath="weights-improvement $-\{$ epoch: $\theta 2 d\}-\{$ val_loss: $-2 f\}$, hdf5

checkpoint = ModelCheckpoint (filepath, monitor $={ }^{\prime}$ val_loss', verbose $=1$, save_best_only=True, mode $=$ "min")

def compare(best, new):
return best.losses ['val_loss'] > new.losses ['val_loss']

def path (new)

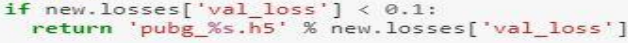

$\mathrm{cb}=[$

utils.GDrivecheckpointer (compare, path)

]

-2018-12-02 20:25:38-. https://raw.githubusercontent.com/Zahlii/colab-tf-utils/master/utils.py 151.101 .1 Resolving raw.githubusercontent.com (raw.githubusercontent.com) ... 151.101.0.133, 151.101.64.133,

Connecting to raw.githubusercontent.com (raw.githubusercontent.com)|151.101.0.133|:443... connected.

HTP rent, awaiting response... 200 OK

Length: 6935 ( 6 .8k)

utils.py.1 $100 \%[=================>] \quad 6.77 \mathrm{~K} \quad \ldots . \mathrm{KB} / \mathrm{s}$ in $0 \mathrm{~s}$

2018-12-02 20:25:38 (85.6 MB/s) - 'utils.py.1' saved [6935/6935]

In [0]: def build_model():

model = Sequential

model.add(Dense(512, kernel_initializer='he_normal", input_dim=x_train.shape[1], activation="relu"))

model.add(BatchNormalization())

model add (Dense 256, kern

(

model. add (Dropout $(0.1)$ )

model.add (Dense(128, kernel_initi

model add (Drop

model add (Dense ( 1 , kernel initializer=" normal" activation= "sigmoid")

optim = optimizers. Adam (1r=0,01, epsilon=1e-8, decay=1e-4, amsgrad=False)

model.compile(optimizer=optim, loss='mse', metrics=['mae'])

\#history = model.fit( $X_{-}$train, $y_{-}$train, epochs=70, batch_size=100000)

return model

In [ө]: downloader $=$ GDrivesync ()

filename=

drive file path=downloader.find items ( filename) [e]

downloader.download_file_to_folder(drive_file_path, filename)

Downloading file pubg 0.007696801999306263 h5 to pubg 0.007696801999306263 . h5: 1008

$[00: 00<00: \theta 0,317,21 i t / s]$

Figure 20: Deep Learning 
International Journal of Engineering Applied Sciences and Technology, 2019

Vol. 3, Issue 12, ISSN No. 2455-2143, Pages 107-118

Published Online April 2019 in IJEAST (http://www.ijeast.com)

In $[\theta]$ : deep.summary ()

\begin{tabular}{|c|c|c|}
\hline Layer (type) & Output Shape & Param \# \\
\hline dense_5 (Dense) & (None, 512) & 22528 \\
\hline batch_normalization_4 (Batch & (None, 512) & 2048 \\
\hline dropout_ 4 (Dropout) & (None, 512) & $\theta$ \\
\hline dense_ 6 (Dense) & (None, 256) & 131328 \\
\hline batch_normalization_5 (Batch & (None, 256) & 1024 \\
\hline dropout_ 5 (Dropout) & (None, 256) & 0 \\
\hline dense_7 (Dense) & (None, 128) & 32896 \\
\hline batch_normalization_6 (Batch & (None, 128) & 512 \\
\hline dropout_6 (Dropout) & (None, 128) & $\theta$ \\
\hline $\begin{array}{l}\text { dense_ } 8 \text { (Dense) } \\
==============0\end{array}$ & (None, 1) & 129 \\
\hline $\begin{array}{l}\text { Total params: } 190,465 \\
\text { Trainable params: } 188,673 \\
\text { Non-trainable params: } 1,792\end{array}$ & & \\
\hline
\end{tabular}

In [0]: deep.load_weights("weights-improvement-06-0.01.hdf5")

In $[\theta]:$ history $=$ deep.fit $(x$ train, y_train,

validation_data $=\left(X_{-} v a l, y_{-} v a 1\right)$,

initial epochs $=30$,

callbacks=cb,

verbose $=1$ )

calculate_error(deep, "Deep")

Train on 3112875 samples, validate on 1334090 samples

$3112875 / 3112875[===========================]$ - 1500s 482us/step - loss: 0.0075 - mean_absolute_error:

0.0636 - val_loss: 0.0085 - val_mean_absolute_error: 0.0657

Epoch 00021 : val_loss did not improve from 0.00811

No improvement.

Epoch $22 / 30$

$3112875 / 3112875[===========================]$ - 1441 s 463 us/step - loss: 0.0075 - mean_absolute_error:

0.0635 - val_loss: 0.0083 - val_mean_absolute_error: 0.0654

Epoch 00022 : val_loss did not improve from 0.00811

No improvement.

Figure 21: Deep Learning Architecture 